\title{
Transient aggregation of particles at interfaces
}

\author{
Antoine Lagarde $\odot,{ }^{1}$ Christophe Josserand, ${ }^{2}$ and Suzie Protière ${ }^{1, *}$ \\ ${ }^{1}$ Institut Jean Le Rond O'Alembert, CNRS, Sorbonne Université, UMR 7190, F-75005 Paris, France \\ ${ }^{2}$ LadHyX, CNRS, Ecole polytechnique, Institut polytechnique de Paris, 91120, Palaiseau, France
}

(Received 5 October 2020; accepted 28 July 2021; published 27 August 2021)

\begin{abstract}
Spherical particles at a liquid interface attract each other and aggregate. The capillary forces at play are well described for a pair of objects, but as soon as many particles interact, no direct calculation is possible and only a statistical approach can account for the global dynamics. Here, we focus on the statistics of clustering of such a system with a long-range interaction which varies during the aggregation process. We measure experimentally and numerically the distribution of sizes as a function of time and exhibit a self-similar clustering mechanism subdivided into two aggregating regimes, with a well-defined transition that we characterize.
\end{abstract}

DOI: 10.1103/PhysRevFluids.6.084307

\section{INTRODUCTION}

Whether they are particles or planets, the motion of two bodies interacting with each other can easily be predicted. However, as soon as one considers more than two objects, statistical tools are needed to investigate their interaction, study their collective dynamics, or characterize their phase ordering and coarsening kinetics [1] (more is different, as stated by Anderson [2]). The resulting complexity leads to the aggregation of systems of all types: from the formation of planets [3-5] to the global order observed in active systems, such as bacteria in suspension [6] that can arrange in large clusters [7], but also between passive objects like vibrated copper-wire segments [8].

This aggregation process can also be observed when particles are placed at a liquid interface at rest: they interact in particular through lateral capillary forces [9], which may then lead to the formation of clusters [10]. In this case the aggregation is two-dimensional and the clustering dynamic is easy to visualize. This capillary interaction is also long-ranged and attractive since it varies on a scale of the order of the capillary length, which is much larger than the particle sizes. Short-ranged forces (such as Van der Waals, or electromagnetic interactions) can be repulsive or attractive and may play an important role in the dynamics near particle coagulation $[11,12]$. As a result of such interactions, submicrometer particles [13] up to millimeter-sized beads [14,15] floating at the surface of a liquid attract one another. Macroscopic objects with well-defined microstructure can then be manufactured using this process [16], either by controlling the shape of the individual objects $[17,18]$ or their surface chemistry [19]. These clustering events has gathered a lot of attention, particularly in the case of small objects subjected to Brownian motion: for instance, nanometer particles moving at an interface can aggregate following their random collisions. In this case, although their individual motion is random, the aggregation process appears to be universal [20,21] and exhibits specific cluster-mass distributions with dynamical scalings [22-25]. However little is known about the aggregation of large and dense particles. In this case, the motion of the particles is deterministic, since Brownian motion can be neglected, and their local capillary

\footnotetext{
"protiere@ida.upmc.fr
} 
deformations, the meniscii due to the gravity-capillary balance, induce long-range interactions [26]. These aspects will necessarily impact the clustering dynamics.

In this paper we characterize the evolution of the cluster-size distribution during the aggregation of interacting granular rafts $[27,28]$. These rafts are formed at a water/oil interface, so that the capillary length $\ell_{c}$ is large $\left(\ell_{c} \approx 10 \mathrm{~mm}\right)$ compared to the air-water one $\left(\ell_{c} \approx 3 \mathrm{~mm}\right)$, and both Van der Waals interactions and electromagnetic forces can be neglected. Furthermore, by contrast with colloidal systems, the action of the weight of the grains on the oil-water interface cannot be resumed to a pointlike force since capillary deformations overlap and produce a global deformation which increases with the raft size [11]. We describe this out-of equilibrium process with a single self-similar function reminiscent of the one observed for the steady-state of active systems, developing some general tools to tackle similar mechanisms. Because the particles are large and dense, the statistical aspect of the clustering dynamics presented here does not come from thermal fluctuations but is due to the random initial configuration, similarly to granular collapse [29]. We expect the process to be different from diffusion-limited aggregation (DLA) [22,30] or colloidal aggregation [21]. Here we show that understanding the aggregation dynamics of such rafts is challenging since their capillary interaction does not obey a simple scaling law. First, it decreases rapidly when the distance between the rafts is larger than the capillary length, similarly to screening in 2D electrostatic potential [12]. Second, the dependance of the interaction force between rafts with the raft sizes makes the usual mean field theory not applicable. Finally, this statistical process is crucial to describe quantitatively the applications of granular rafts through oil encapsulation and sinking $[27,28]$.

\section{EXPERIMENTS}

A typical experiment is displayed in Fig. 1(a) (Movie 1 showing the mechanism provided as Supplemental Material [31]). A thick layer of silicone oil (kinematic viscosity $v_{o}=100 \times 10^{-6} \mathrm{~m}^{2} \mathrm{~s}^{-1}$, density $\rho_{o}=966 \mathrm{kgm}^{-3}$, oil-water surface tension $\gamma=42 \mathrm{mNm}^{-1}$ ) is poured into a tank of dimensions $0.3 \times 0.3 \times 0.25 \mathrm{~m}$ filled with deionized water (density $\rho_{w}=1000 \mathrm{kgm}^{-3}$ ). A specific number of ceramic particles $n_{\text {tot }}$ of density $\rho_{\text {part }}=3,800 \mathrm{kgm}^{-3}$ and radius $R_{\text {part }}=0.125 \mathrm{~mm} \pm$ $25 \mu \mathrm{m}$ is then randomly sprinkled above the oil layer, inside a circular domain of radius $R_{\text {domain }}$. We make sure that the initial spatial distribution of particles at the interface is homogeneous. The particles travel through the oil layer and then get trapped at the oil-water interface where they start to interact. The capillary length is $\ell_{c}=\sqrt{\frac{\gamma}{\left(\rho_{w}-\rho_{o}\right) g}} \approx 10 \mathrm{~mm}$. Each experiment is performed five times for every pair of parameters $\left(n_{\text {tot }}, R_{\text {domain }}\right)$ to average the measurements between the five clustering processes, so that the initial positions of the particles do not impact the statistical results. The parameters explored in the experiments are $123 \leqslant n_{\text {tot }} \leqslant 915$, with $2 \mathrm{~cm} \leqslant R_{\text {domain }} \leqslant 5.15 \mathrm{~cm}$. To prevent the particles from interacting with the tank's walls, we must limit the maximum size of the domain to $5.15 \mathrm{~cm}$. We can easily track the particles on the liquid interface since they contrast with the liquid bath and form circular objects when they are imaged from the top. We measure the area of each cluster of particles as it varies over time and deduce from it the number of particles in each cluster. With this method the deformation of the interface is neglected but the particles are very small and we make check that the total number of particles does not vary with time during each experiment.

When the aggregation starts, the particles are spread out at the interface. After $1000 \mathrm{~s}$, clusters begin to form and continue to grow as they attract all the remaining single particles. These clusters finally merge until all particles are collected into a single granular raft. We show the distribution of cluster sizes at three successive times on Fig. 1(c) in red circles: the $x$ axis presents the size of a cluster, while in ordinate the corresponding average number of clusters $N(n, t)$ divided by $n_{\text {tot }}$ is displayed. Although this distribution function is not normalized [the probability for a grain to be in a cluster of size $n$ is in fact $n N(n, t) / n_{\text {tot }}$ ], this notation is usually preferred to characterize the aggregation dynamics in colloidal systems as it enables the rescaling of the functions according to the Schmoluchowski equation [20]. The error bars are due both to the average obtained by 


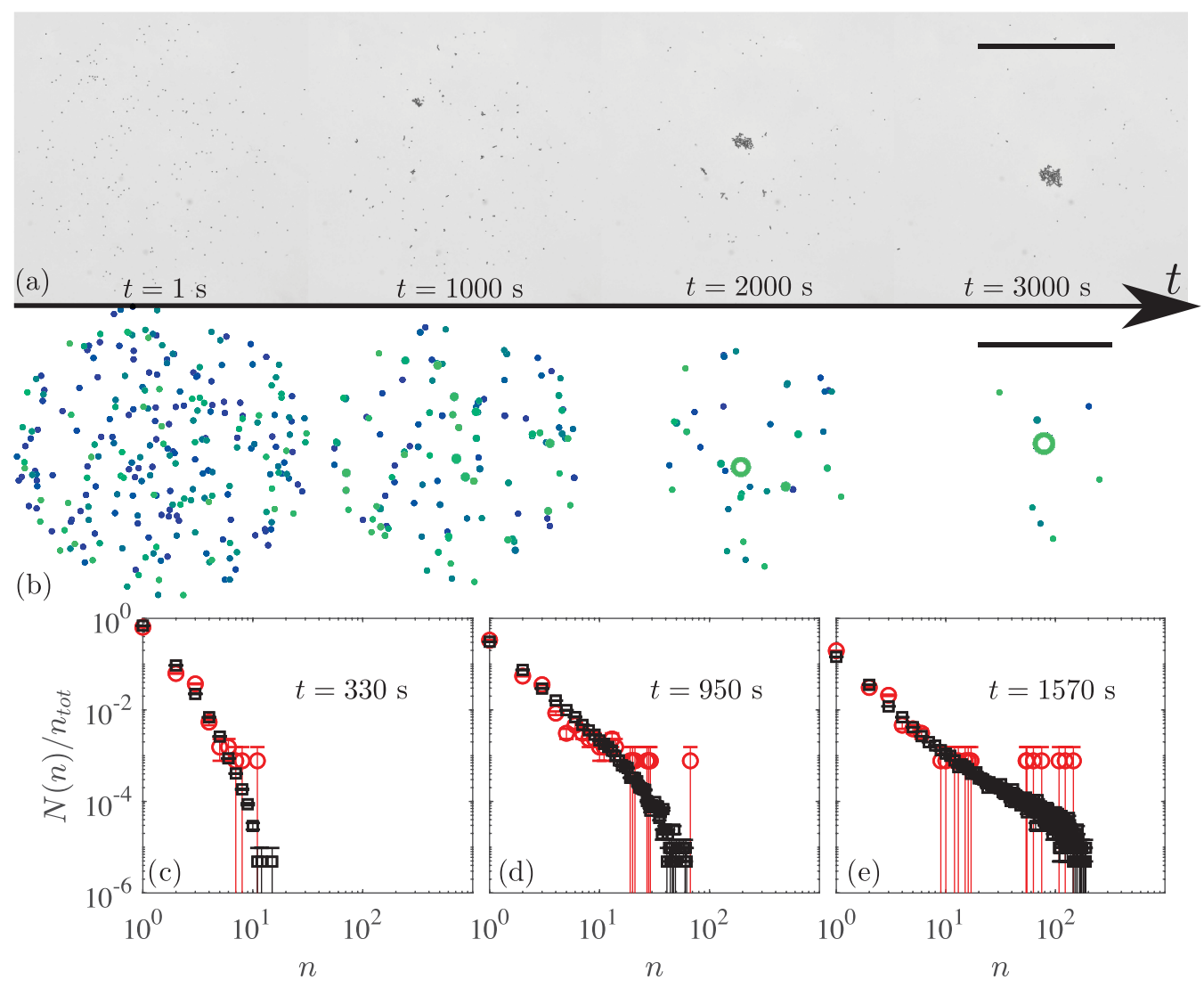

FIG. 1. Experimental and numerical aggregation. Comparison between the aggregation of particles at an interface from an experimental perspective in panel (a) and a numerical one in panel (b) $\left(n_{\text {tot }}=248, R_{\text {domain }}=\right.$ $3.25 \mathrm{~cm}$ in both cases). Scale bars: $3 \mathrm{~cm}$. The colors in panel (b) are used to distinguish the different rafts. In panels (c), (d), and (e), we plot the distribution of sizes at three different time steps. The results are obtained by averaging the distributions of 5 experiments (red circles) and 890 simulations (black squares). The error bars are calculated as the minimum number of rafts we can detect: $1 /\left(n_{\text {tot }} N_{\text {simulations }}\right)$.

performing here five experiments for each set of parameters (the more experiments presented, the more precise the distribution will be) and the finite size (and thus finite number of grains $n_{\text {tot }}$ ) of the experiments. We now focus on the evolution of the distribution. Initially, the particles are mostly isolated. During the clustering process, the number of isolated particles appears to be monotonically decreasing as larger clusters appear: $N(n, t) / n_{\text {tot }}$ transits from a sharp left-centered distribution to a more scattered one when the clusters become larger. The dynamics exhibit an irreversible process leading to the formation of increasingly large clusters as time increases. No equilibrium can be reached until all the particles are gathered in a single raft for finite-size domains, while the cluster distribution should widen indefinitely for infinite domains.

\section{NUMERICAL SIMULATIONS}

Due to experimental limitations, the distributions are always truncated at large $n$. To overcome this problem, we performed numerical simulations of this same process. To simulate the aggregation of $n_{\text {tot }}$ particles randomly distributed inside a circle of radius $R_{\text {domain }}$ we base our model on the results from our previous work [32]. We compute the equilibrium position of a bead at an interface, 
by deriving the capillary interaction between a pair of identical beads at a fluid-liquid interface as follows [14]:

$$
\begin{aligned}
\overrightarrow{F_{\text {cap } 1 \rightarrow 1}} & =C_{1} K_{1}\left(L / \ell_{c}\right) \vec{e}, \\
\overrightarrow{F_{\text {drag } 1}} & =-C_{2} V G^{-1}\left(\frac{L}{R_{\text {part }}}\right) \vec{e},
\end{aligned}
$$

with $\vec{e}$ the unitary vector directed from one particle to the other (along the direction of motion), $\ell_{c}$ the capillary length, $C_{1}$ and $C_{2}$ coefficients that depend on the fluid and particle parameters [32], $V$ the speed of the particle considered, $K_{1}$ the modified Bessel function of the second kind of order 1 , and $R_{\text {part }}$ the radius of the particles. $L$ is the distance between the centers of the two rafts while $G$ is the mobility function that accounts for the drainage of the liquid between the particles as they get closer [33]. $\overrightarrow{F_{\text {cap } 1 \rightarrow 1}}$ is the capillary force between single particles, and $\overrightarrow{F_{\text {drag }} 1}$ the drag experienced by a single particle.

This result is then generalized by looking for the capillary and drag forces between two granular rafts [32]. These forces can be derived from their expressions for single particles, multiplied by coefficients we wish to determine and that, we assume, depends on the number of particles in each raft:

$$
\begin{aligned}
\overrightarrow{F_{\text {cap } B \rightarrow A}} & =f\left(n_{A}, n_{B}\right) \overrightarrow{F_{\text {cap } 1 \rightarrow 1}}, \\
\overrightarrow{F_{\text {drag } A}} & =g\left(n_{A}\right) \overrightarrow{F_{\text {drag }} 1} .
\end{aligned}
$$

Previous experiments have then allowed us to determine the expression of $f\left(n_{A}, n_{B}\right)$ and $g\left(n_{A}\right)$ :

$$
\begin{aligned}
\overrightarrow{F_{\text {cap } B \rightarrow A}} & =n_{A} n_{B} C_{1} K_{1}\left(L / \ell_{c}\right) \overrightarrow{e_{A \rightarrow B}}, \\
\overrightarrow{F_{\text {drag } A}} & =-\sqrt{n_{A}} C_{2} V_{A} G^{-1}\left(\frac{l+2 R_{\text {part }}}{R_{\text {part }}}\right) \overrightarrow{e_{A \rightarrow B}},
\end{aligned}
$$

with $\overrightarrow{e_{A \rightarrow B}}$ the unitary vector directed from $\mathrm{A}$ to $\mathrm{B}$ (along the direction of motion), $n_{A}, n_{B}$ the number of particles in each raft, $V_{A}$ the speed of raft A. $l$ is the distance between the two closest particles from each raft. This leads to the following velocity vector for a raft A attracted by a raft B:

$$
V_{A} \overrightarrow{e_{A \rightarrow B}}=\sqrt{n_{A}} n_{B} \frac{C_{1}}{C_{2}} G\left(\frac{l+2 R_{\text {part }}}{R_{\text {part }}}\right) K_{1}\left(\frac{L}{\ell_{c}}\right) \overrightarrow{e_{A \rightarrow B}} .
$$

This model has some limitations. Since it uses linearized equations in its derivation, it becomes less valid when the deformation of the interface increases. In the present work, we use small particles compared to Ref. [32] (here, $R_{\text {part }}=0.125 \mathrm{~mm}$ ). In the range explored, the vertical deformation imposed by the granular rafts on the interface thus always remains in the linear limit. Another limitation concerns the Reynolds number, which can be larger than 1 for large clusters. Here, for all the experiments and all the simulations, the Reynolds number remains very small compared to one. Finally, the previous expression gives us the total velocity along the interface. Yet, the oil-water interface does not remain completely planar, since each raft deflects it downwards. Because we are going to perform a two-dimensional simulation, we therefore need to compute the horizontal projection of this total velocity.

We computed the geometry of the interface (Ref. [32] and its Supplemental Material). In the linear limit, the slope of the interface at a distance $L$ from the raft is the solution of the following system:

$$
\begin{aligned}
\nabla^{2} h & =\frac{h}{\ell_{c}^{2}}, \\
h(r \rightarrow \infty) & =0, \\
h\left(r=R_{\text {raft }}\right) & =h_{\text {raft }},
\end{aligned}
$$


with $h_{\text {raft }}$, the depth of the interface at the edge of the raft and $h$ the vertical axis corresponding to the height of the undisturbed flat interface. The solution of this equation can then be written as follows:

$$
\frac{d h}{d L}=\frac{h_{\text {raft }} / \ell_{c}}{K_{0}\left(R_{\text {raft }} / \ell_{c}\right)} K_{1}\left(\frac{L}{\ell_{c}}\right),
$$

with $K_{0}$ the modified Bessel function of the second kind of order $0, h_{\text {raft }}$ the depth of the edge of the raft, and $R_{\text {raft }}$ the radius of the raft. In the Supplemental Material of Ref. [32], we have also developed a minimal model to express the ratio $\frac{h_{\text {ratt }} / \ell_{c}}{K_{0}\left(R_{\text {raft }} / \ell_{c}\right)}$, that turns out to be linear in the number of particles:

$$
\frac{h_{\text {raft }} / \ell_{c}}{K_{0}\left(R_{\mathrm{raft}} / \ell_{c}\right)}=C_{3} n
$$

with $n$ the number of particles inside the raft, and $C_{3}$ a coefficient depending on the fluid and particle properties (see Ref. [32] for more details).

We can now compute the value of $d h / d L$ for any raft sizes. The horizontal velocity of a raft made of $n_{i}$ particles, attracted by a raft of size $n_{j}$, is then expressed simply as:

$$
V_{i}^{(x)}(i, j) \overrightarrow{e_{i j}}=\sqrt{n_{i}} n_{j} \frac{C_{1}}{C_{2}} G\left(\frac{l_{i j}+2 R_{\text {part }}}{R_{\text {part }}}\right) K_{1}\left(\frac{L_{i j}}{\ell_{c}}\right) \cos \left\{\tan ^{-1}\left[C_{3} n_{j} K_{1}\left(\frac{L_{i j}}{\ell_{c}}\right)\right]\right\} \overrightarrow{e_{i j}},
$$

with $\overrightarrow{e_{i j}}$ the unitary vector along the direction connecting the rafts $i$ and $j$, directed toward $j, L_{i j}$ the distance between raft $i$ and $j$, and $l_{i j}$ the distance between their edge. From Eq. (8), we can compute the respective velocities of two interacting rafts. But we aim to simulate many rafts, each one interacting with all the others. This is where we make our major hypothesis: we assume that all forces can be superimposed. This means that we can calculate $V_{i}^{(x)}(i, j)$ for each pair of rafts, and then sum all the different velocities. The velocity vector of a raft of size $i$ is therefore

$$
\overrightarrow{V_{i}^{(x)}}(i)=\sum_{j=1}^{n_{\text {clusters }}} V_{i}^{(x)}(i, j) \overrightarrow{e_{i j}}
$$

We could have chosen to only take into account the contribution from the closest neighbor since there may be little interaction between rafts that are far apart. However, the capillary force evolves with the interraft distance according to a modified Bessel function of the second kind of order one, as described above, which decreases exponentially at large distances (large with respect to the capillary length). As a consequence, this exponential decrease makes the contributions of the rafts far from the considered object completely negligible. This other possibility would not have changed the final results.

This calculation is performed for all the different rafts at each time step, and then the position of each raft is moved accordingly. We use an adaptive time step defined as follows:

$$
\Delta t=\max \left(\frac{R_{\mathrm{part}}}{\max _{i} V_{i}^{(x)}}, \frac{\min _{i, j} L_{i j}}{10 \max _{i} V_{i}^{(x)}}\right) .
$$

The first term of the max in Eq. (10) ensures that even the fastest raft will never travel by more than the radius of one particle, while the second one allows bigger time steps when all particles are very far from one another. Using this adaptive time step, we end up with simulations that last a few hours when several hundred of particles are simulated. The code is written in Matlab, and can perfectly run on a personal laptop. Since we needed to run a lot of simulations to have statistics, the code was launched on machines Intel Xeon CPU E5-2658 v2 @ $2.40 \mathrm{GHz}$ with 32 Gb RAM.

Since we do not consider the specific rearrangements of the particles inside the clusters and only in the aggregation dynamics, we automatically merge two rafts of sizes $n_{i}$ and $n_{j}$ and radius $\sqrt{n_{i}+n_{j}} R_{\text {part }}$ when they touch one another (we neglect the fact that particles inside a raft do not 
cover $100 \%$ of the area of the raft). We computed the interacting forces between two rafts $i$ and $j$, consisting of respectively $n_{i}$ and $n_{j}$ particles, as a function of the forces between two individual particles:

$$
\begin{aligned}
F_{\text {cap } j \rightarrow i} & =n_{i} n_{j} F_{\text {cap } 1 \rightarrow 1}, \\
F_{\text {drag } i} & =\sqrt{n_{i}} F_{\text {drag } 1},
\end{aligned}
$$

where $F_{\text {cap } j \rightarrow i}$ and $F_{\text {drag } i}$ are the capillary and drag forces experienced by raft $i$, and $F_{\text {cap } 1 \rightarrow 1}$ and $F_{\text {drag } 1}$ the same forces but for a pair of individual beads. These two forces can be calculated as functions of the interparticle distances as well as the fluids and beads properties [14]. The dependencies of each total force with the radius of the clusters are characterized experimentally in Ref. [32]. They can be described by considering that the meniscus deformation is influenced by the total number of particles and that the drag is only sensitive to the radius of the cluster (by analogy with Stokes' drag law). The Reynolds number, based on the viscosity of the oil layer, the radius of the raft and its speed, is very small, ( $\mathrm{Re} \leqslant 5 \times 10^{-2}$ for all rafts at all times) so that the drag is simply proportional to the velocity. We write the equilibrium between the two forces Eqs. (11a) and (11b) to compute the velocity of a raft $i$ attracted by another raft $j, V_{i}(i, j)$. We then assume that all forces can be superimposed. We can now calculate $V_{i}(i, j)$ for each pair of rafts and then sum all the different velocity contributions. The velocity vector of a raft of size $i$ is then

$$
\vec{V}_{i}(i)=\sum_{j} V_{i}(i, j) \overrightarrow{e_{i j}}
$$

with $\overrightarrow{e_{i j}}$ the unitary vector along the direction connecting the rafts $i$ and $j$, directed toward $j$.

Starting from an initially random distribution of particles inside a circular domain of radius $R_{\text {domain }}$, we calculate the velocity of each raft at every time step. Once two rafts are in contact, they form a new cluster and we have them merge numerically.

\section{CLUSTERING DYNAMICS}

A typical simulation is displayed in Fig. 1(b) (Supplemental Material provides the corresponding movie, Movie 2 [31]). The aggregation follows the same steps as in the experiments, within the same time scales: a scattered distribution of isolated particles, then a few larger rafts appear until they merge into a single large cluster that attracts all the remaining particles. These similarities are quantitatively confirmed by the measurement of the distributions, as emphasized in Figs. 1(c)-1(e), where the two curves are superimposed. Our simulations reproduce the clustering dynamics of the experiments without any adjustable parameter, indicating that our model contains the pertinent physical mechanisms. Moreover, the simulations compensate for the poor statistics of the experiments: in particular, the error bars at the lower boundary for $N(n) / n_{\text {tot }}$ decreases by two orders of magnitude in the simulation, which gives access to the whole function instead of just a portion.

The distributions evolve irreversibly with time and, for a given time, two regimes can be observed [Fig. 1(d) with a transition at $n \approx 30$, for example]. To understand more extensively the shape of these distributions we now analyze the numerical results as they provide greater statistics for a complete study. We investigate the distributions' dependence both with $n$ and $t$, as represented in Fig. 2. The distributions during the final times can be discarded, since at this point only a single raft attracts all the other particles and the finite size of our system starts to play a role. We focus on the distributions up to the time at which the biggest cluster has collected $60 \%$ of all particles [dotted line in Fig. 2(d)]. At a fixed $t, N(n, t)$ is a decreasing function of $n$. This decrease will not remain true throughout the whole clustering for finite size domains since at the end, all probabilities of existence will equal zero except for the biggest raft containing all the particles. Two regimes can then be identified: for low values of $n, N(n, t)$ decreases with a slope close to $n^{-1.5}$, and for large values of $n$, the decrease steepens. The position $[n, N(n, t)]$ of the transition between these two regimes also seems to vary with time. Here again, a transition can be identified for each curve. We 

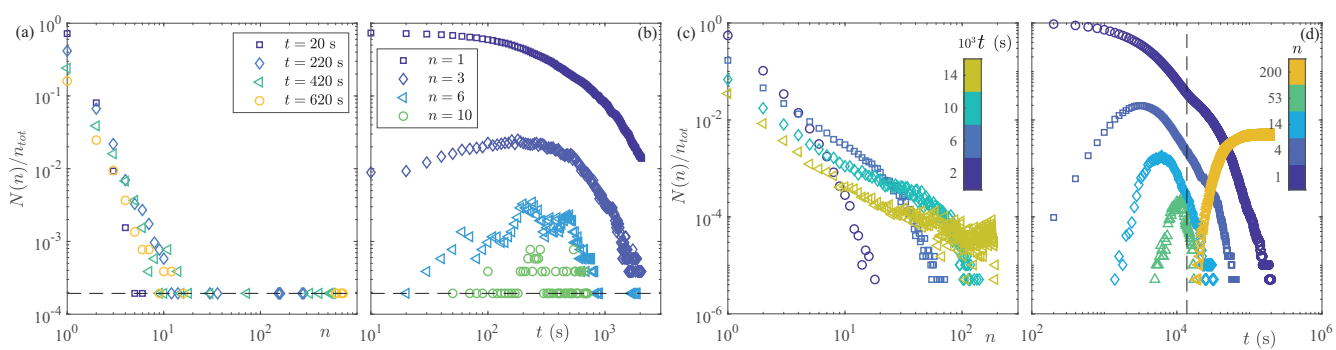

FIG. 2. Distribution of sizes. Evolution off the distributions of sizes as a function of the two variables $n$ and $t$. Experiments in panels (a) and (b): In panel (a), the distributions are plotted as a function of $n$, for 4 successive times after the beginning of the clustering, and as a function of $t$ for four different raft radii in panel (b). The data come from the averaging of five experiments with 968 particles inside a domain of radius $R_{\text {domain }}=3.25 \mathrm{~cm}$. The horizontal dotted line corresponds to the lowest possible value of $N(n)$. Simulations in panels (c) and (d): In panel (c), the distributions are plotted as a function of $n$, for four successive times after the beginning of the clustering (dark blue circles: $t=2000 \mathrm{~s}$, light blue squares: $t=6000 \mathrm{~s}$, green diamonds: $t=10000 \mathrm{~s}$, yellow triangles: $t=14000 \mathrm{~s}$ ), and as a function of $t$ for five different raft radii in panel (d) (dark blue circles: $n=1$, blue squares: $n=4$, light blue diamonds: $n=14$, green up-pointing Triangle: $n=53$, orange left-pointing triangle: $n=200)$. The data come from the averaging of 980 simulations $\left(n_{\text {tot }}=200\right.$, $R_{\text {domain }}=5.8 \mathrm{~cm}$ ). The dotted line in d represents the maximum time up to which we look at the distributions in panel (c). Error bars are not plotted for clarity reasons.

then conducted experiments and simulations when varying the number of particles and the sizes of the domains. The same type of distribution with the same regimes and transitions is observed. We can thus compare these results with their experimental counterparts [Fig. 2(a) and 2(b)]. Similarly to the simulations, we recover that at a fixed $t, N(n)$ is a decreasing function of $n$. In contrast to the simulations, the existence of the two decaying regimes is far from being clear. This is due to the poor statistics of the experiments (since for a given set of parameters, each experiment is done only five times). However, we can still observe a faster decrease for $t=20 \mathrm{~s}$ than for larger times. Focusing now on Figs. 2(b) and 2(d), we can look at the behavior of $N$ for fixed values of $n$. Here again, the same behavior is found both in the simulations and the experiments: as expected, $N(1, t)$ is a monotonically decreasing function of time. For the other curves, $N(n, t)$ has a nonmonotonic evolution, with first an increase of its value up to a maximum, followed by a decrease.

\section{SELF-SIMILARITY}

In many similar aggregating systems, as described in the Introduction, the goal is to find a selfsimilar function able to capture the clustering mechanisms, which can be commonly described by the Smoluchowski coagulation equations [34,35]. We thus rescale our data using the moment of order 2 of the distributions:

$$
P_{2}=\frac{\sum_{j=1}^{\infty} j^{2} N(j, t)}{n_{\mathrm{tot}}} ;
$$

since $j N(j, t) / n_{\text {tot }}$ is the probability of a grain to be in a cluster of size $j, P_{2}$ can be understood as a measure of the mean cluster size. We now look for a self-similar solution in the form of Eq. (14):

$$
\frac{N(n, t)}{n_{\mathrm{tot}}}=P_{2}(t)^{-2} \psi\left[\frac{n}{P_{2}(t)}\right],
$$

with $\psi$ the self-similar function we are seeking. The corresponding result is plotted in Fig. 3 . The numerical and experimental rescalings collapse relatively well onto the same curve. The few experimental points which do not collapse onto the master curve are due to the same issue we 


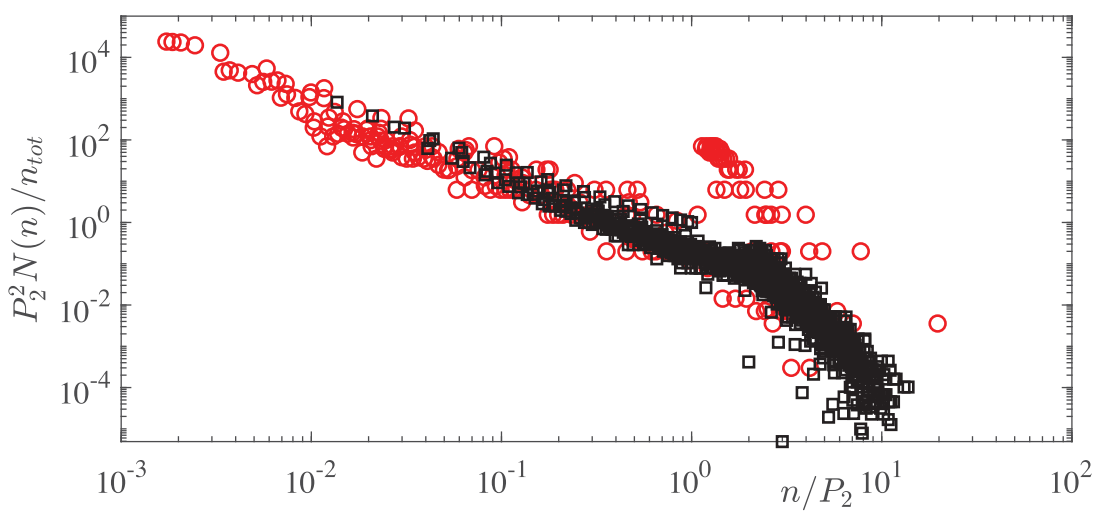

FIG. 3. Comparison of the rescaling of the distribution of sizes for an experiment in red $\left(n_{\mathrm{tot}}=915\right.$, $\left.R_{\text {domain }}=5.15 \mathrm{~cm}\right)$, and a simulation in black $\left(n_{\mathrm{tot}}=300, R_{\text {domain }}=2.9 \mathrm{~cm}\right)$.

encountered previously: the number of experiments and the relatively small size of the clusters we could make for each experiments leads to low statistics. However, all the data collapse onto the same curve without any adjustable parameters. This gives us once again the reassurance that our simulations capture the aggregation process perfectly. Therefore, we can focus on the simulations (Fig. 4). The rescaling captures the time dependency of the clustering, leading to the collapse of all the data for all times and the localization of the transition into a single point. The two regimes can now be characterized more precisely, by a first decrease with a power law close to -1.5 and a second regime that can be fitted by a -5 power law which could be in fact the signature of either this power law or an exponential decay. The transition between these two regimes is located at $n / P_{2} \approx 1$, and the fit (red curve in Fig. 4) gives the approximated self-similar function $\psi$ :

$$
\psi(x)=x^{-1.2} \exp (-x)
$$

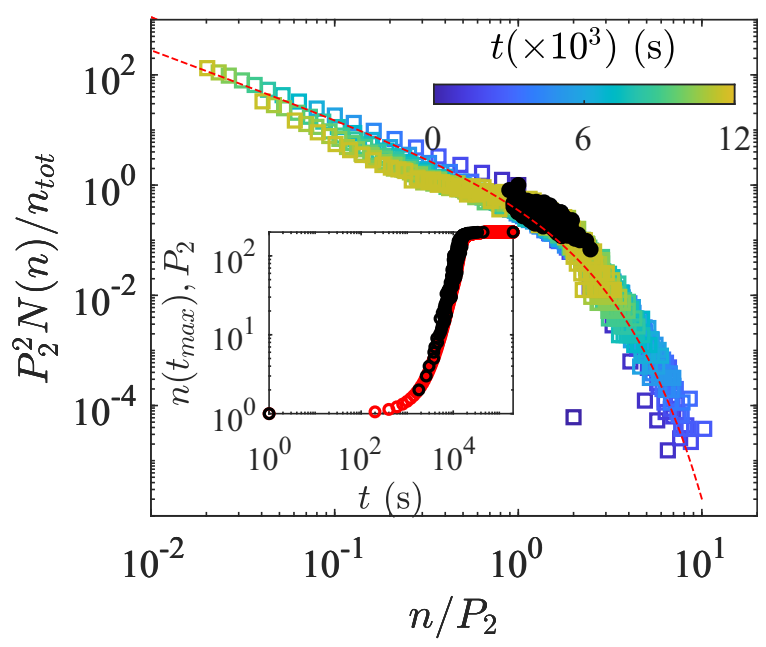

FIG. 4. Rescaling of the distributions Distribution of sizes rescaled according to Eq. (14). The data come from the averaging of 980 simulations $\left(n_{\text {tot }}=200, R_{\text {domain }}=5.8 \mathrm{~cm}\right)$. In black dots, we plot the rescaled positions of the maxima of the distributions with respect to time, for each value of $n$. In red, the fit corresponding to Eq. (15). The color bar represents the time, in a linear scale. Inset: Evolution of the moment of order $2 P_{2}$ in red, compared to $n\left(t_{\max }\right)$ in black. Error bars are not plotted for clarity reasons. 
In the range of parameters explored, this self-similar function does not depend on the initial number of particles, the size of the domain, or the initial surface density. We also identify the existence of a maximum of the distributions for each size of cluster [Figs. 2(b) and 2(d)]. Each maximum is described by a triplet $\left(n, t_{\max }, N_{\max }\right)$, which can then be inserted in the rescaled distributions (black dots, Fig. 4). The number of clusters of size $n$ is maximum (and equal to $N_{\max }$ ) at $t_{\max }$ : it increases before and decreases after $t_{\max }$. All these maxima are localized right at the transition between the two regimes. This result provides a strong physical meaning to the clustering regimes observed. For large $n / P_{2}$, the rafts are created faster than they are disappearing while for small $n / P_{2}$, they disappear by merging with other particles faster than they are formed. The transition corresponds to the equilibrium between creation and disappearance of specific rafts, but also to the maximum probability of existence of a given raft size throughout the whole clustering process. The smaller clusters are attracted to the larger ones that are slower moving, suggesting why different scaling laws for the cluster distributions should be expected.

The transition is localized near $n / P_{2} \approx 1$. The evolution with time of both $n\left(t_{\max }\right)$ and $P_{2}(t)$ (inset Fig. 4) shows that they are almost identical and do not exhibit clear power or exponential laws. This indicates that the time evolution of the cluster distribution is totally enclosed in that of $P_{2}(t)$. Keeping in mind that $P_{2}$ measures the mean cluster size, this transition separates the smaller clusters that will eventually be absorbed by the larger ones.

\section{CONCLUSIONS}

The regimes obtained here differ from what was observed in other passive aggregating systems, such as DLA (constant regime for small $n$ for 2D aggregation) or reaction-limited aggregation (one decaying regime with a single slope) [21,30,36]. A similar cluster-size distribution, with a power-law decay for small sizes (with a smaller slope than the one observed in our system) and an exponential one for large sizes, is found for the steady-state of self-propelled objects [37-39] or bacterial suspensions $[7,40]$. However, in those cases, the distribution results from the equilibrium between aggregation and escapes of the individual objects. In the system we investigate here, no equilibrium, no erosion, and only passive interactions are observed. As time goes on, the power-law distribution, valid for small $n$ (compared to $P_{2}$ ), spreads over larger and larger raft sizes [since $P_{2}(t)$ monotonically increases with time]. This suggests that the raft distribution obeys a self-similar mechanism of front propagation with two different regimes depending on whether the raft size is after [for $n<n\left(t_{\max }\right) \sim P_{2}(t)$ ] or before [for $n>n\left(t_{\max }\right) \sim P_{2}(t)$ ] the front.

To conclude, we have investigated the aggregation of particles at a liquid-fluid interface, from a disperse initial condition into a single large cluster. This aggregation of granular rafts is driven by capillary interactions and the passive beads aggregate following a self-similar mechanism that was previously only observed for the steady-state of active systems. The self-similar function governing the mechanism follows two successive regimes, depending on the rate of creation of a given raft compared to its merging with other clusters, and an equilibrium at which the transition occurs. Although the nature of the two regimes and their respective behavior remain yet to be explained, granular rafts provides a new system to investigate the statistical theory of aggregation and better understand clustering dynamics in general.

[1] A. J. Bray, Theory of phase-ordering kinetics, Adv. Phys. 51, 481 (2002).

[2] P. W. Anderson, More is different, Science 177, 393 (1972).

[3] M. H. Lee, On the validity of the coagulation equation and the nature of runaway growth, Icarus 143,74 (2000).

[4] T. Steinpilz, K. Joeris, F. Jungmann, D. Wolf, L. Brendel, J. Teiser, T. Shinbrot, and G. Wurm, Electrical charging overcomes the bouncing barrier in planet formation, Nat. Phys. 16, 225 (2020). 
[5] J. Blum and G. Wurm, The growth mechanisms of macroscopic bodies in protoplanetary disks, Annu. Rev. Astron. Astrophys. 46, 21 (2008).

[6] H.-P. Zhang, A. Be'er, E.-L. Florin, and H. L. Swinney, Collective motion and density fluctuations in bacterial colonies, Proc. Natl. Acad. Sci. U.S.A. 107, 13626 (2010).

[7] F. Peruani, J. Starruß, V. Jakovljevic, L. Søgaard-Andersen, A. Deutsch, and M. Bär, Collective Motion and Nonequilibrium Cluster Formation in Colonies of Gliding Bacteria, Phys. Rev. Lett. 108, 098102 (2012).

[8] V. Narayan, S. Ramaswamy, and N. Menon, Long-lived giant number fluctuations in a swarming granular nematic, Science 317, 105 (2007).

[9] P. A. Kralchevsky and N. D. Denkov, Capillary forces and structuring in layers of colloid particles, Curr. Opin. Colloid Interface Sci. 6, 383 (2001).

[10] J. Bleibel, S. Dietrich, A. Domìnguez, and M. Oettel, Shock Waves in Capillary Collapse of Colloids: A Model System for Two-Dimensional Screened Newtonian Gravity, Phys. Rev. Lett. 107, 128302 (2011).

[11] A. Vincze, A. Agod, J. Kertész, M. Zrínyi, and Z. Hórvölgyi, Aggregation kinetics in two dimensions: Real experiments and computer simulations, J. Chem. Phys. 114, 520 (2001).

[12] A. Domìnguez, M. Oettel, and S. Dietrich, Dynamics of colloidal particles with capillary interactions, Phys. Rev. E 82, 011402 (2010).

[13] P. A. Kralchevsky, N. D. Denkov, and K. D. Danov, Particles with an undulated contact line at a fluid interface: Interaction between capillary quadrupoles and rheology of particulate monolayers, Langmuir 17, 7694 (2001).

[14] D. Vella and L. Mahadevan, The cheerios effect, Am. J. Phys. 73, 817 (2005).

[15] M. M. Nicolson, The interaction between floating particles, Proc. Cambridge Philosoph. Soc. 45, 288 (1949).

[16] U. Srinivasan, D. Liepmann, and R. T. Howe, Microstructure to substrate self-assembly using capillary forces, J. Microelectromech. Syst. 10, 17 (2001).

[17] L. Botto, E. P. Lewandowski, M. Cavallaro, and K. J. Stebe, Capillary interactions between anisotropic particles, Soft Matter 8, 9957 (2012).

[18] J.-C. Loudet, A. M. Alsayed, J. Zhang, and A. G. Yodh, Capillary Interactions Between Anisotropic Colloidal Particles, Phys. Rev. Lett. 94, 018301 (2005).

[19] C. Casagrande, P. Fabre, E. Raphael, and M. Veyssié, Janus beads: Realization and behaviour at water-oil interfaces, Europhys. Lett. 9, 251 (1989).

[20] M. Y. Lin, H. M. Lindsay, D. A. Weitz, R. C. Ball, R. Klein, and P. Meakin, Universality in colloid aggregation, Nature (London) 339, 360 (1989).

[21] D. A. Weitz and M. Y. Lin, Dynamic Scaling of Cluster-Mass Distributions in Kinetic Colloid Aggregation, Phys. Rev. Lett. 57, 2037 (1986).

[22] T. A. Witten and L. M. Sander, Diffusion-Limited Aggregation, a Kinetic Critical Phenomenon, Phys. Rev. Lett. 47, 1400 (1981).

[23] T. A. Witten and L. M. Sander, Diffusion-limited aggregation, Phys. Rev. B 27, 5686 (1983).

[24] W. D. Brown and R. C. Ball, Computer simulation of chemically limited aggregation, J. Phys. A: Math. Gen. 18, L517 (1985).

[25] P. Meakin and F. Family, Structure and dynamics of reaction-limited aggregation, Phys. Rev. A 36, 5498 (1987).

[26] J. Bush and D. Hu, Walking on water: Biolocomotion at the interface, Annu. Rev. Fluid Mech. 38, 339 (2006).

[27] M. Abkarian, S. Protière, J. M. Aristoff, and H. A. Stone, Gravity-induced encapsulation of liquids by destabilization of granular rafts, Nat. Commun. 4, 1895 (2013).

[28] S. Protière, C. Josserand, J. M. Aristoff, H. A. Stone, and M. Abkarian, Sinking a Granular Raft, Phys. Rev. Lett. 118, 108001 (2017).

[29] I. Goldhirsch and G. Zanetti, Clustering instability in dissipative gases, Phys. Rev. Lett 70, 1619 (1993).

[30] I. Jullien, R. Botet, and P. Mors, Computer simulations of cluster-cluster aggregation, Faraday Discuss. Chem. Soc. 83, 125 (1987). 
[31] See Supplemental Material at http://link.aps.org/supplemental/10.1103/PhysRevFluids.6.084307 for experimental and numerical videos of the described aggregation mechanism.

[32] A. Lagarde, C. Josserand, and S. Protière, The capillary interaction between pairs of granular rafts, Soft Matter 15, 5695 (2019).

[33] N. D. Vassileva, D. van den Ende, F. Mugele, and J. Mellema, Capillary forces between spherical particles floating at a liquid-liquid interface, Langmuir 21, 11190 (2005).

[34] M. V. Smoluchowski, Drei Vorträge über Diffusion, Brownsche MolekularBewegung und Koagulation von Kolloidteilchen, Physik. Zeit. 17, 557 (1916).

[35] D. J. Aldous, Deterministic and stochastic models for coalescence (aggregation and coagulation): A review of the mean-field theory for probabilists, Bernoulli 5, 3 (1999).

[36] A. González, F. Marínez-López, A. Moncho-Jordá, and R. Hidalgo-Álvarez, Concentration effects on two- and three-dimensional colloidal aggregation, Physica A 314, 235 (2002).

[37] D. Levis and L. Berthier, Clustering and heterogeneous dynamics in a kinetic Monte Carlo model of self-propelled hard disks, Phys. Rev. E 89, 062301 (2014).

[38] G. S. Redner, C. G. Wagner, A. Baskaran, and M. F. Hagan, Classical Nucleation Theory Description of Active Colloid Assembly, Phys. Rev. Lett. 117, 148002 (2016).

[39] Y. Yang, V. Marceau, and G. Gompper, Swarm behavior of self-propelled rods and swimming flagella, Phys. Rev. E 82, 031904 (2010).

[40] R. Soto and R. Golestanian, Run-and-tumble dynamics in a crowded environment: Persistent exclusion process for swimmers, Phys. Rev. E 89, 012706 (2014). 